\title{
Ultrasound Biosafety Considerations for the Practicing Sonographer and Sonologist
}

Thomas R. Nelson, PhD, J. Brian Fowlkes, PhD, Jacques S. Abramowicz, MD, Charles C. Church, PhD

\begin{abstract}
Abbreviations
ALARA, as low as reasonably achievable; FDA, Food and Drug Administration; $I_{\text {SPTA }}$, spatial-peak temporal-average intensity; MI, mechanical index; ODS, output display standard; TI, thermal index; TIB, thermal index for bone; $\mathrm{TIC}$, thermal index for cranial bone; TIS, thermal index for soft tissue
\end{abstract}

Received October 16, 2008, from the Departments of Radiology and Bioengineering, University of California, San Diego, La Jolla, California USA (T.R.N.); Departments of Radiology and Bioengineering, University of Michigan, Ann Arbor, Michigan USA (J.B.F.); Department of Obstetrics and Gynecology, Rush University, Chicago, Illinois USA (J.S.A.); and National Center for Physical Acoustics, University of Mississippi, University, Mississippi USA (C.C.C.). Revision requested October 17, 2008. Revised manuscript accepted for publication November 7, 2008.

We thank Karen J. Ophir, BS, RDMS, Dolores $H$. Pretorius, MD, Marvin C. Ziskin, MD, and Gerald R. Harris, PhD, for helpful suggestions.

Address correspondence to Thomas R. Nelson, PhD, Department of Radiology, University of California, San Diego, Medical Teaching Facility, Room 113, 9500 Gilman Dr, La Jolla, CA 92093-0610 USA.

E-mail: tnelson@ucsd.edu

Article includes CME test

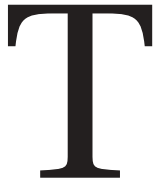

he purpose of this article is to present the practicing sonographer and sonologist with an overview of the biohazards of ultrasound and guidelines for safe use. Key words: bioeffects; fetal; mechanical index; output display standard; thermal index; ultrasound.

\section{Overview}

Ultrasound is an imaging modality that has important diagnostic value. Although useful in a variety of applications, diagnostic ultrasound is particularly useful in prenatal diagnosis. To date, there is no evidence that diagnostic ultrasound produces harm in humans or the developing fetus when used properly.

There are, however, an increasing range of ultrasound studies being performed. Newer technologies can have higher acoustic output levels than earlier equipment. Also, subtle or transient effects of diagnostic ultrasound, such as changes in membrane permeability or neuronal migration, are not completely understood.

Therefore, diagnostic ultrasound should be used prudently with ultrasound examinations performed only by trained, competent personnel. To ensure continued safety, it is essential to maintain an awareness of the potential for bioeffects, especially with newer equipment and more sophisticated procedures.

The purpose of this article is to review ultrasound biosafety considerations for the practicing sonographer and sonologist as well as to provide references for more detailed discussion and guidance. There has been considerable debate within the ultrasound bioeffects community regarding setting specific values for the mechanical index (MI) and thermal index (TI) to limit bioeffects risk. There also has been some reluctance in the bioeffects community to help sonographers and sonologists make appropriate practical clinical biosafety decisions because of this debate. 
This article should be viewed as the considered opinion of the authors and should not be taken as the position of any professional society or medical specialty board. This article is an effort on our part to start a discussion on what constitutes appropriate practical guidance to the sonographer and sonologist regarding acoustic output values consistent with obtaining diagnostic information while protecting patient welfare.

\section{Ultrasound Acoustic Output and Bioeffects}

Some tissues, such as those in the developing embryo and fetus, are particularly sensitive to energy deposition from many imaging modalities, including ultrasound. Up to 8 weeks after conception, organogenesis is taking place in the embryo. This is a period when cell damage might lead to anomalies or subtle developmental changes. Fetal effects also vary with the increasing mineralization of developing bone, raising the potential for heating of sensitive tissues such as brain and spinal cord, although the increasing fetal size in later gestation may provide some additional resistance to thermal insults. The brain and spinal cord continue to develop through to the neonatal period, so continued vigilance is necessary.

The presence of bone within the ultrasound beam greatly increases the likelihood of a temperature rise due to direct absorption in the bone itself and conduction of heat from bone to adjacent tissues. Generally, the temperature rise is greatest at bone surfaces and the adjacent soft tissues. As a result, it also is important to minimize eye exposures in the fetus and adult because of the relatively low perfusion in the eye, particularly in the lens, which has a reduced capability for heat dissipation.

Furthermore, because ultrasound is mechanical energy, the mechanical interaction of sound with cells and tissues has the potential to produce nonthermal bioeffects.

Limited information is available regarding possible subtle biological effects at diagnostic levels in fetal, pediatric, and adult patients. Therefore, one should limit acoustic output and exposure time commensurate with obtaining an acceptable diagnostic evaluation. At present, there is no reason to withhold diagnostic scanning, includ- ing during pregnancy, provided it is medically indicated and is used prudently by fully trained operators. ${ }^{1-3}$ However, it also is important to keep in mind that the potential for bioeffects also exists with equipment that is not adjusted properly or used prudently.

\section{Standard for Real-time Display of Thermal and Mechanical Acoustic Output Indices on Diagnostic Ultrasound Equipment}

Before 1976, there were no limits to the permissible acoustic output from diagnostic ultrasound equipment. In 1976, the US Food and Drug Administration (FDA) began regulating medical devices, including ultrasound, and eventually developed 4 application-specific exposure limits (peripheral vascular, $720 \mathrm{~mW} / \mathrm{cm}^{2}$; cardiac, 430 $\mathrm{mW} / \mathrm{cm}^{2}$; fetal and other, $94 \mathrm{~mW} / \mathrm{cm}^{2}$; and ophthalmic, $\left.17 \mathrm{~mW} / \mathrm{cm}^{2}\right){ }^{4,5}$ This regulatory output level was established on the basis of the predicate devices in use in the market at that time and the apparent safety of ultrasound as understood at that time.

In 1992, at the request of manufacturers and end users interested in obtaining specific improvements in the diagnostic capabilities of ultrasound, the FDA changed this limit to 720 $\mathrm{mW} / \mathrm{cm}^{2}$ for all applications except eye scanning. Most prenatal epidemiologic studies on bioeffects have been based on ultrasound exposures occurring before 1992 with equipment regulated according to the pre-1992 output limits of $94 \mathrm{~mW} / \mathrm{cm}^{2}$. Along with the change in output limits, the FDA also mandated that machines capable of producing higher outputs be able to display to the diagnostician some indication of the relative potential for ultrasound-induced bioeffects. This regulation is known as the Standard for Real-Time Display of Thermal and Mechanical Acoustic Output Indices on Diagnostic Ultrasound Equipment, more commonly known as the output display standard (ODS). ${ }^{4,6}$

The original proposal for implementation of the ODS was to remove limits on machine output, placing full responsibility on the operator. Subsequently, it was decided to implement the ODS with the limits along with some indication of the acoustic output on the display. Since 1992, equipment is capable of operating with acoustic 
outputs of up to $720 \mathrm{~mW} / \mathrm{cm}^{2}$ with the specific acoustic output under the direct control of the operator and with the expectation that techniques that are as low as reasonably achievable (ALARA) will be used.

The ODS consists of the MI and the TI. The MI is an on-screen indicator of the relative potential for ultrasound to induce an adverse bioeffect by a nonthermal mechanism. The TI is an onscreen indicator of the relative potential for a tissue temperature rise. The TI is a model-based approach to determine where the maximum temperature and location in the acoustic field would be expected on the basis of the imaging parameters and the acoustic propagation model selected.

Although not perfect, TI and MI estimates represent the most practical approach to thermal and nonthermal risk estimation currently available. Nevertheless, for the acoustic indices to be meaningful, the diagnostician must be familiar with ultrasound safety issues and their implications for fetal and patient imaging studies. Implementation of the ODS puts much greater responsibility for patient safety on the ultrasound end user. Adherence to the ALARA principle also is recommended.

An additional major requirement for acceptance of the ODS by the FDA pertains to adequate education of end users. This requires information about the ODS be provided by the manufacturer, which is most commonly in the form of the publication Medical Ultrasound Safety ${ }^{7}$ from the American Institute of Ultrasound in Medicine, which is provided with new ultrasound scanners. However, the broader goal for sonographers and sonologists should be an understanding of the potential for bioeffects through initial applications training by the vendor, ongoing continuing medical education courses, and vigilant daily awareness of acoustic output.

\section{Acoustic Physics}

\section{Physical Properties of Ultrasound}

Ultrasound is mechanical energy that propagates longitudinally through elastic media, such as tissues, creating alternating zones of compression and rarefaction. Ultrasound imaging typically uses short pulses (Figure 1) with acoustic energy reflected back toward the transducer from interfaces having different acoustic properties. Typical biomedical ultrasound imaging parameters are shown in Table $1 .^{8}$

The acoustic power is the rate of energy production. The Système International d'Unités unit of power is the watt $(1 \mathrm{~J} / \mathrm{s})$. The acoustic intensity is the rate of energy flowing through a unit area $^{6}$ (watts per square centimeter). Acoustic output is measured for a variety of pulse conditions with the intensity relationships between the various measured parameters shown in Table 2 and Figure 1.

The current FDA output limits for diagnostic ultrasound are a spatial-peak temporal-average intensity $\left(\mathrm{I}_{\mathrm{SPTA}}\right)$ less than $720 \mathrm{~mW} / \mathrm{cm}^{2}$. The acoustic output depends on the output power, pulse repetition frequency, and scanner operating mode (eg, B-mode, M-mode, pulsed, or color or power Doppler imaging). ${ }^{4,9}$

Figure 1. Acoustic pulses showing where and how acoustic output is measured and reported for a single scan line at the focus of a diagnostic medical ultrasound system.

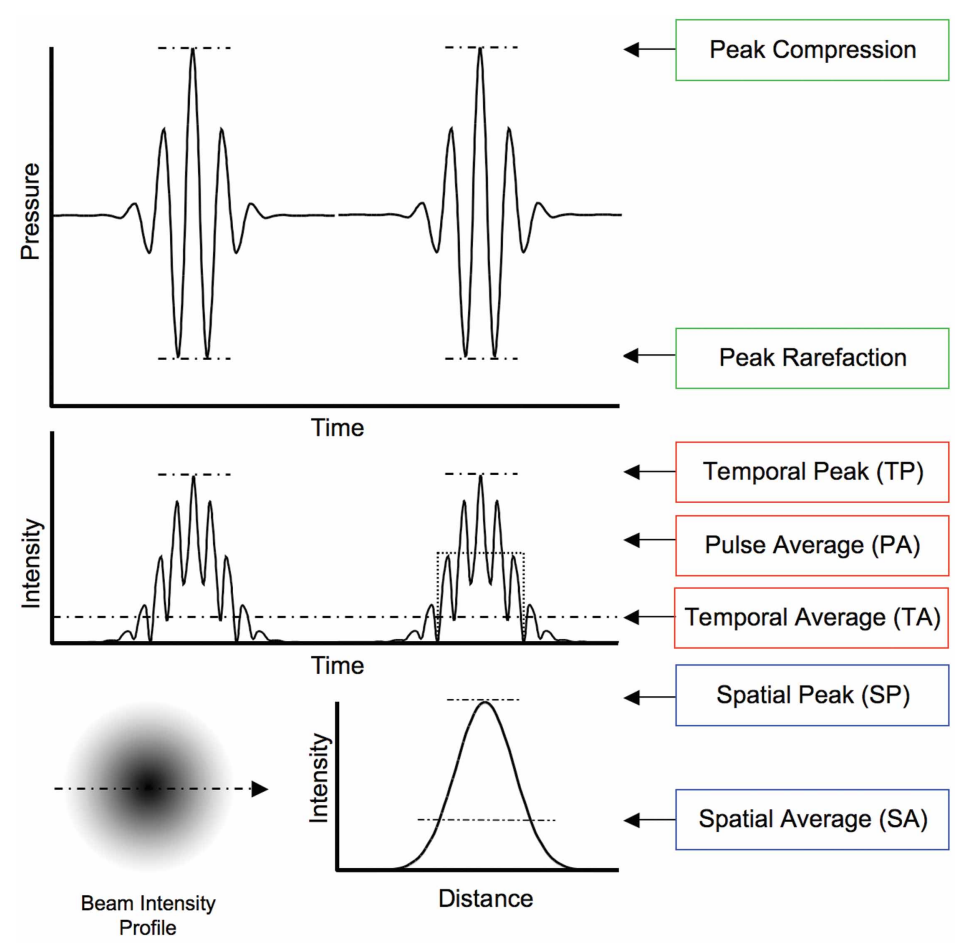


Table 1. Diagnostic Medical Ultrasound Properties

\begin{tabular}{ll}
\hline Parameter & \multicolumn{1}{c}{ Value } \\
\hline Speed of sound & $1.45-2.1 \mathrm{~mm} / \mu \mathrm{s}^{\mathrm{a}}$ \\
Frequency & $1-15 \mathrm{MHz}$ \\
Wavelength & $0.1-1.5 \mathrm{~mm}$ \\
Pulse length & $3-5 \mathrm{cycles}$ \\
Attenuation & $0.3 \mathrm{~dB} /(\mathrm{cm}-\mathrm{MHz})$ (derated) \\
Frame rate & up to $150 \mathrm{frames} / \mathrm{second}$ (30 frames/second typical) \\
\hline
\end{tabular}

aNominal scanner values are typically set to $1.54 \mathrm{~mm} / \mu \mathrm{s}$.

\section{Mechanical Index}

The MI is intended to offer a guide to the likelihood of a nonthermal bioeffect, including cavitation, and is related to the intensity of the pulse. ${ }^{10-18}$ The MI is defined as the maximum value of the peak negative pressure divided by the square root of the acoustic center frequency. The MI is based on the derated peak rarefactional pressure.*

Cavitation is the phenomenon wherein bubbles form in a liquid material when the local pressure (such as might be produced by the rarefaction part of a passing ultrasound wave) falls below the vapor pressure of the liquid sufficient to pull the material apart. Cavitation generally falls into 2 types: (1) inertial or transient cavitation, in which the newly formed bubble rapidly collapses, producing a shock wave that can be capable of biological effects; and (2) noninertial cavitation, in which the bubble oscillates in the acoustic field and appears to be less likely to produce biological effects.

Ultrasound contrast agents also can play a role in cavitation. Ultrasound contrast agents typically are stable gas-filled microbubbles. Although cavitation can occur without ultrasound contrast agents, the presence of microbubbles can increase the probability of cavitation and other nonthermal effects. ${ }^{2,19-21}$ Animal studies have shown that ultrasound contrast agents potentially can enhance cavitation and microstreaming effects, resulting in microvascular damage or capillary rupture..$^{20,21}$
Ultrasound contrast agents also have been associated with induction of premature ventricular contractions in echocardiography using high-MI pulses. Appropriately triggered excitation can be used to reduce the occurrence of such effects. In general, the risk of cavitation increases with an increasing MI. ${ }^{3}$

The potential for ultrasound nonthermal biohazards exists if equipment is used imprudently. Damage has been shown in animal models for tissues having gas pockets at MI values greater than 0.4. Thus, one should avoid unnecessary exposure to tissues such as the neonatal lung. As a general strategy, the MI should be kept as low as possible while obtaining the necessary diagnostic information.

\section{Thermal Index}

The TI is defined as the ratio of the emitted acoustic power to the power required to raise the temperature of tissue by $1^{\circ} \mathrm{C}$. The $\mathrm{TI}$ is intended to give a rough guide to the magnitude of the temperature rise that might be produced after a long exposure, although technically the TI is not a measure of the precise temperature rise. ${ }^{22,23}$ A larger TI value represents a higher heating potential and correspondingly a higher risk (eg, a TI of 2 means a higher risk than a TI of 1.5 but not as high a risk as a TI of 3 ).

There are 3 forms of the TI that may be displayed, according to the application:

1. The thermal index for soft tissue (TIS). This assumes that the ultrasound beam does not impinge on bone but only insonates soft tissue, such as in the first trimester.

2. The thermal index for bone (TIB). This assumes that the beam impinges on bone at or near its focus and where appropriate should be displayed in the second and third trimesters.

3. The thermal index for cranial bone (TIC). This assumes that the transducer front face is very close to bone, such as when scanning in neonatal, pediatric, and adult patients.

\footnotetext{
* The maximum value of the peak negative pressure anywhere in the ultrasound field measured in water is reduced by an attenuation factor equal to that which would be produced by a medium having an attenuation coefficient of $0.3 \mathrm{~dB} /(\mathrm{cm}-\mathrm{MHz})$ typically referred to as a process called derating. All of the FDA regulatory levels are specified with derated $\mathrm{I}_{\mathrm{SPTA}}$ values.
} 
Table 2. Acoustic Intensity Output Measurement Parameters

\begin{tabular}{|c|c|c|}
\hline Parameter & Symbol & Description \\
\hline Spatial-peak temporal-peak & $\mathrm{I}_{\text {SPTP }}$ & $\begin{array}{l}\text { The highest intensity measured at any point in the ultrasound } \\
\text { beam and at any time; it is the highest value of the measured } \\
\text { intensities (more closely related to potential mechanical bioef- } \\
\text { fects and cavitation) }\end{array}$ \\
\hline Spatial-peak pulse-average & $\mathrm{I}_{\text {SPPA }}$ & $\begin{array}{l}\text { The highest intensity measured at any point in the ultrasound } \\
\text { beam averaged over the temporal (time) duration of the pulse }\end{array}$ \\
\hline Spatial-peak temporal-average & $\mathrm{I}_{\text {SPTA }}$ & $\begin{array}{l}\text { The highest intensity measured at any point in the ultrasound } \\
\text { beam averaged over the pulse repetition period (more closely } \\
\text { related to the magnitude of thermal bioeffects) }\end{array}$ \\
\hline Spatial-average temporal-peak & $I_{\text {SATP }}$ & $\begin{array}{l}\text { The average intensity over a selected area, such as the transducer } \\
\text { face, but at the peak in time }\end{array}$ \\
\hline Spatial-average pulse-average & $\mathrm{I}_{\text {SAPA }}$ & $\begin{array}{l}\text { The average intensity over a selected area, such as the transducer } \\
\text { face, averaged over the temporal duration of pulse }\end{array}$ \\
\hline Spatial-average temporal-average & $\mathrm{I}_{\text {SATA }}$ & $\begin{array}{l}\text { The average intensity over a selected area, such as the transducer } \\
\text { face, averaged over the pulse repetition period; this measure- } \\
\text { ment of intensity is frequently quoted and is the lowest value of } \\
\text { the measures of intensity }\end{array}$ \\
\hline
\end{tabular}

Definitions are given approximately in order of decreasing intensity value for the same pulse conditions.

The maximum temperature and its location in the acoustic field for the TIS and TIB would be at the focal point of the ultrasound beam, but such is not necessarily the case for the TIC. However, limitations of the simple models on which the TI is based mean that TI values may underestimate the temperature elevation by a factor of 2 or greater, although more typically, the TI overestimates the temperature. ${ }^{2}$

A temperature elevation less than $1.5^{\circ} \mathrm{Clikely}$ does not present a bioeffects risk to the embryo, although there has been some debate on the threshold nature of thermal effects. ${ }^{24} \mathrm{~A}$ temperature elevation greater than $4^{\circ} \mathrm{C}$ for 5 minutes can present a bioeffects risk to the embryo. ${ }^{25}$ Velocity, power, and pulsed spectral Doppler ultrasound all have the potential to reach these levels. The TI provides a guide for the sonographer and sonologist regarding the magnitude of the temperature increase.

\section{Acoustic Reporting Requirements}

The acoustic reporting requirements are implemented by the FDA, which uses a 2-track approach to marketing clearance: tracks 1 and 3. Track 1 is for devices that do not follow the ODS and therefore have application-specific output limits; track 3 is for devices that conform to the ODS. There is no longer a track 2.

Systems that include fetal Doppler applications, except for fetal heart rate monitors, should follow track 3 . Track 3 does not apply to systems for which a display would be required but that have fixed acoustic output.

Under track 3, acoustic output limits are not determined on an application-specific basis, but the global maximum derated $\mathrm{I}_{\text {SPTA }}$ must be 720 $\mathrm{mW} / \mathrm{cm}^{2}$ or less, and either the global maximum MI must be 1.9 or less or the global maximum derated spatial-peak pulse average intensity must be $190 \mathrm{~W} / \mathrm{cm}^{2}$ or less. An exception is for ophthalmic use, in which case the TI = $\max$ (TIS, TIC) and is not to exceed 1 ; the $\mathrm{I}_{\text {SPTA.3 }}$ should be 50 $\mathrm{mW} / \mathrm{cm}^{2}$ or less; and the MI is not to exceed 0.23 .

The displayed TI and MI values are updated by the machine as control settings are changed by the operator (Figure 2).

\section{Guidelines for Safe Use}

\section{Medical Endorsement}

Diagnostic ultrasound equipment should only be used for medical diagnosis. Ultrasound equipment should only be used by persons who are fully trained in the safe and proper operation of the equipment (see "Appendix" for a description of FDA-recommended educational program content). The operator should have a full awareness of machine settings and understand the effect of those machine settings on thermal and mechanical bioeffects. The initial power setting and scanner default protocols should be set for lower power. Exposure times and power levels should follow the ALARA principle during scanning. 


\section{Thermal and Mechanical Indices}

As a general strategy, the on-screen TI and MI values should be monitored after scanner adjustments and kept ALARA. Although there is some variation in opinions within the ultrasound bioeffects community regarding the relationship between acoustic output and acoustic bioeffects, one might consider the following as suggested guidelines for setting and monitoring acoustic output during scanning.

\section{Prenatal Examinations}

1. Thermal index values less than 0.5 should be used unless otherwise required, particularly in the first trimester.

2. More generally, TI values less than 0.5 likely can be used for scanning times on an extended basis.

3. Thermal index values greater than 0.5 and up to 1 should be limited to scanning times less than 30 minutes.

4. Thermal index values greater than 2.5 should be limited to scanning times less than 1 minute.

5. Mechanical index values less than 0.4 should be used if gas bodies may be present.

6. In the absence of gas bodies, MI values can be increased as needed but should remain low because mechanisms for bioeffects not related to gas bodies may be possible in the developing fetus.

\section{Postnatal Examinations}

1. Thermal index values less than 2 likely can be used for scanning times on an extended basis.

2. Thermal index values greater than 2 and up to 6 should be limited to scanning times less than 30 minutes.

3. Thermal index values greater than 6 should be limited to scanning times less than 1 minute.

4. Mechanical index values less than 0.4 should be used if gas bodies may be present.

5. In the absence of gas bodies, MI values up to 1.9 (current FDA limit) can be used as needed.

6. For neonatal studies, one should consider further limiting exposure levels and durations as developmental processes are continuing.

Although these are suggested guidelines for setting and monitoring acoustic output during scanning, it is understood that depending on the scanning conditions and diagnostic requirements of the study, these levels may be exceeded

Figure 2. Examples of $\mathrm{Ml}$ and $\mathrm{TI}$ displays on ultrasound images from various manufacturers (images courtesy of $\mathrm{D}$. Pretorius, MD, University of California, San Diego).
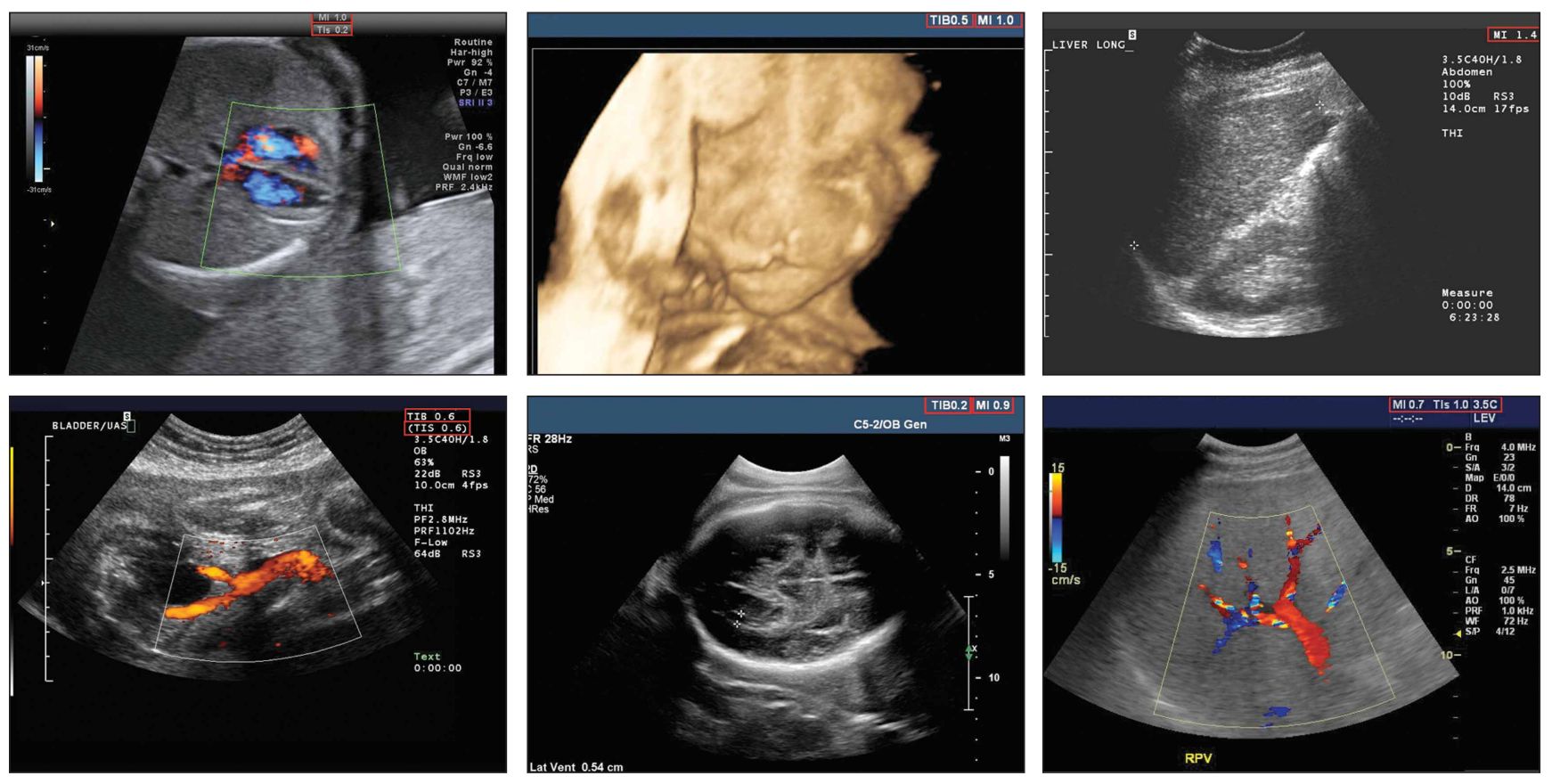

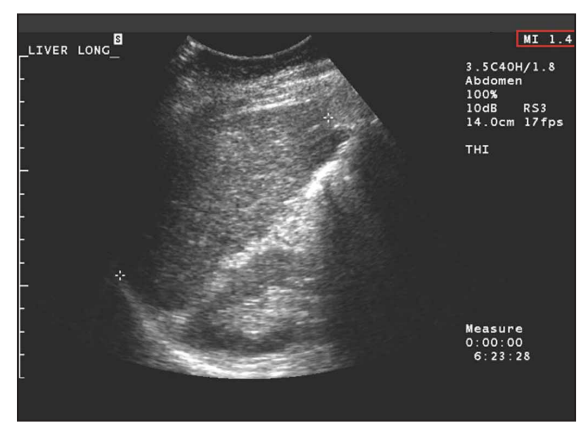


for limited periods to obtain diagnostically appropriate information and to ensure optimal patient care.

Some nonimaging procedures, such as using Doppler ultrasound for fetal heart monitoring or peripheral pulse monitoring, use both continuous wave and pulsed low-power output levels that can cause tissue effects, although the exact mechanisms are still not completely understood. Low-intensity pulsed ultrasound effects, such as bone fracture repair reported in humans and repair of soft tissue damage and accelerated nerve regeneration in animal models, indicate a need for further research on potential fetal effects (Stratmeyer et al, ${ }^{20}$ page 602).

In distinction to other medical imaging modalities in which increases in imaging device output generally improve image quality, it is worth keeping in mind that the relationship between the ultrasonic acoustic output, MI, and TI and image and data quality is not always as straightforward (Figure 3). Thus, always beginning with low MI and TI values is a good overall strategy, only increasing power as the needs of the study dictate.

For fetal scanning, the TIS should be used during the first 8 weeks after conception, with the TIB being used after 8 weeks. In the fetus and adult, the TIS should be used for eye scanning. In neonatal, pediatric, and adult patients, the TIC should be used when the ultrasound field is close to bone in the skull, such as with transcranial Doppler ultrasound, which uses higher power levels; otherwise, the TIB should be used for everything else.
Special care and reduced scanning times should be used for sensitive tissues such as those found in the embryo ( $<8$ weeks), eye, head, brain, and spine. In general, prolonged pulsed Doppler ultrasound is not recommended for these sensitive tissues. ${ }^{2}$ The presence of a preexisting temperature elevation, such as with elevated maternal temperatures, should be considered with regard to minimizing scan times, as should the potential for probe self-heating. ${ }^{10}$

Finally, when the probe is held in a stationary position, the freeze-frame (ie, nonimaging) mode should be used.

\section{Nondiagnostic Imaging Applications}

Nondiagnostic uses of ultrasound equipment, such as repeated scans of healthy subjects for equipment demonstrations generally should be avoided. First-trimester fetal scans should not use color and power Doppler modes and should not be performed for the sole purpose of producing souvenir videos or photographs.

Production of fetal souvenir photographs or videos during diagnostic clinical studies should not increase exposure levels or extend scan times beyond those needed for clinical purposes. Operators should follow safe scanning guidelines and ALARA principles.

The FDA considers the promotion, selling, or leasing of ultrasound equipment for making "keepsake fetal videos" that are not part of a medical diagnostic procedure to be an unapproved use of a medical device. Thus, the use of a

Figure 3. Study in same patient (fetal age, 29 weeks) using different Ml and TI values. Note that the overall study quality is not dramatically affected by the difference in $\mathrm{Ml}$ and $\mathrm{TI}$ values, emphasizing the importance of beginning with lower acoustic output and only increasing as necessary.
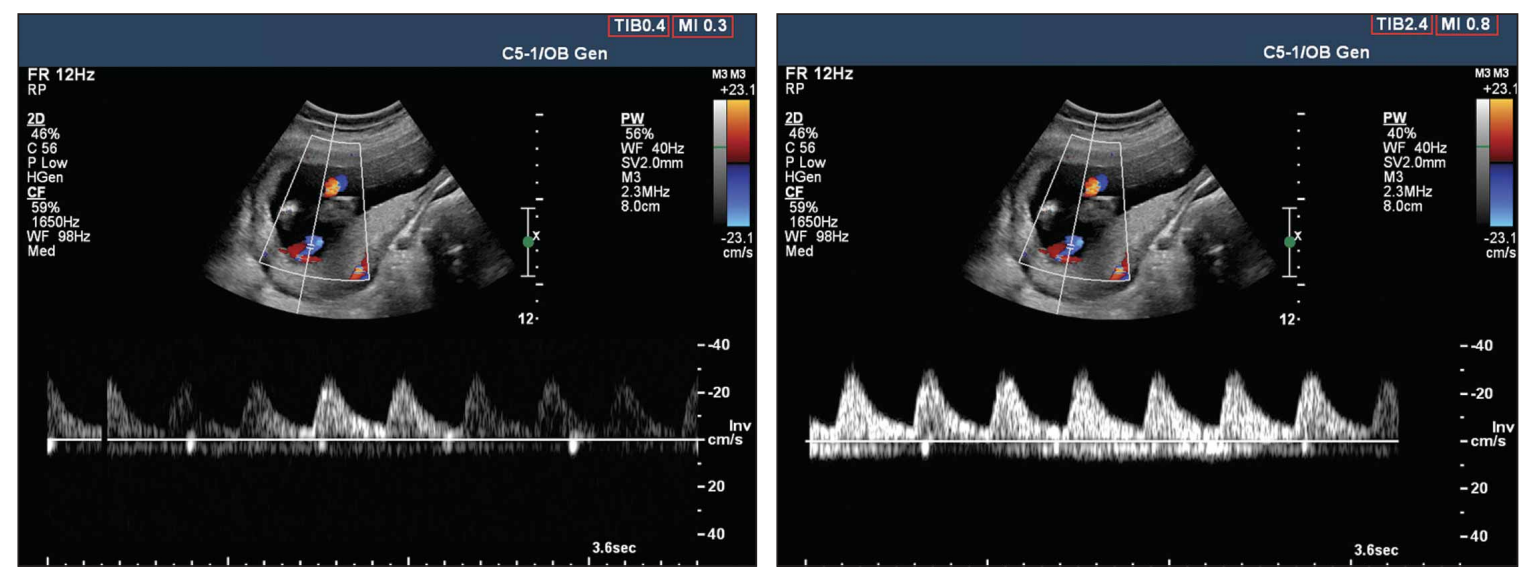
diagnostic ultrasound system for these purposes, without a physician's order, may be in violation of state laws or regulations. ${ }^{26}$

For ultrasound scanner training purposes, the subject should be informed as to how the ultrasound scan relates to typical diagnostic studies. During training sessions, frequent or extended exposure of the same subject is to be avoided, and the acoustic output should be kept as low as possible. $^{2}$

\section{Conclusions}

Technological advances in ultrasound imaging equipment provide improved visualization, diagnosis, and management. Such improvements have substantially improved fetal, pediatric, and adult patient imaging, especially prenatal management.

It is important to keep in mind that diagnostic ultrasound transmits energy into the patient. Diagnostic ultrasound studies of adult, pediatric, and fetal patients generally are considered safe. However, diagnostic procedures should be performed only when there is a valid medical indication.

Most diagnostic ultrasound procedures are performed in the range of the guidelines suggested here. For example, ultrasonic output levels during routine obstetric ultrasound examinations as reflected by the displayed MI and TI vales are generally low. ${ }^{27}$ However, higher output levels, particularly TI levels greater than 1.5, can be achieved, especially with Doppler ultrasound, requiring extra diligence even though they may account for only a very small part of the examination time. ${ }^{28}$

More recent ultrasound scanning technology, such as 3-dimensional sonography, can provide clearer images of patient anatomy such as the developing fetus that are recognizable to the family and physician. Four-dimensional sonographic equipment further facilitates observing movements similar to real-time 2-dimensional imaging. Such capability potentially invites longer viewing times, especially in the fetus. ${ }^{29}$ At present, 3-dimensional sonography likely does not introduce additional safety considerations, although 4-dimensional sonography with continuous exposure offers the potential to prolong examination times and thus increase the potential for bioeffects. ${ }^{3}$
Patient scanning with ultrasound should use the lowest possible acoustic output setting to obtain the necessary diagnostic information under the ALARA principle. The reader is referred to a series of recent articles in the Journal of Ultrasound in Medicine that provide an in-depth review of ultrasound bioeffects and exposimetry. ${ }^{19-23,30,31}$

Education of sonographers and sonologists is key to maintaining a safe and productive ultrasound scanning environment for patients. Unfortunately, recent reports ${ }^{32}$ suggest that most ultrasound users are poorly informed regarding ultrasound safety issues, including the presence and location of MI and TI information on the scanner display. Further efforts in improving the education and training of the ultrasound community are needed to increase end user knowledge of the acoustic output of the machines and safety issues (see "Appendix").

\section{Summary}

The absence of human studies showing ultrasound bioeffects leaves us to pose the question of why we should be concerned about an as yet to be shown potential for bioeffects from diagnostic ultrasound. Fundamentally, the lack of an observed effect does not implicitly suggest that there is no effect but only implies that we have not been able, for whatever reason, to detect one. This, although good news, does not remove the responsibility all sonographers and sonologists have to actively advocate for their patients' well-being by understanding the proper use of their ultrasound scanners and biosafety considerations.

What is known at present is that ultrasound bioeffects have been shown in animal models under acoustic output conditions similar to those used in humans. ${ }^{10-23,30,31}$ We do not explicitly know how the results of these studies relate to humans, but because these studies raise the possibility of effects, it is important to conduct diagnostic ultrasound patient studies as safely as possible while we continue to gain additional information and insight through basic and clinical research.

Diagnostic ultrasound equipment enhances the care and treatment of patients and thus provides a valuable and safe contribution to improving health care when used in a thoughtful and appropriate manner. Sonographers and sonologists have the ultimate responsibility for the safe use of diagnostic ultrasound equipment. 


\section{Appendix: US Food and Drug Administration Education Program on Ultrasound Systems Using the ODS (Track 3) 5}

\subsection{Track 3: Education Program}

1. 3.4.1. Provide an ALARA education program for the clinical end user that covers the subjects listed below; ALARA is an acronym for the principle of prudent use of diagnostic ultrasound by obtaining the diagnostic information at an output that is as low as reasonably achievable. This education program should include explanations of the following:

a. The basic interaction between ultrasound and matter;

b. The possible biological effects;

c. The derivation and meaning of the indices;

d. A recommendation to use and the need to follow the ALARA principle in all studies;

e. Clinical examples of specific applications of the ALARA principle;

f. A document published by the American Institute of Ultrasound in Medicine, Medical Ultrasound Safety (new document forthcoming), which is acceptable to the FDA as meeting the generic content of the educational program. The manufacturer also should provide information specific to its device regarding ALARA.

2. 3.4.2. Minimum requirements for educational material for track 3 devices:

a. 3.4.2.1. Bioeffects and biophysics of ultrasound interactions:

i. Brief description of ultrasound, diagnostic frequencies, and energy levels;

ii. Brief description of the change in policy that requires user education;

iii. Short history of ultrasound use and safety record;

iv. Potential hazards at high output levels;

v. Biological effect mechanisms: thermal, mechanical;

vi. Exposure-effect studies (range of outputs);

vii. Risk versus benefit;

viii. Present state of output levels: higher than historical levels;

ix. Proposed indices as indicators of thermal and mechanical effects.

b. 3.4.2.2. Thermal mechanisms:

i. Description of thermal bioeffects: temperature rise;

ii. Tissue type (soft, bone, fluid) and relative absorption;

iii. Transducer type (frequency, focusing) and relationship to exposure;

iv. Attenuation, absorption, and scattering mechanisms in different tissue types;

v. Spatial volume of insonified tissue (at focus or elsewhere):

A. Homogeneity of tissue in an insonified volume (effects of layering);

B. Soft tissue;

C. Bone tissue (fetal, skull, other);

D. Fluids and gas.

c. 3.4.2.3. Nonthermal mechanisms:

i. Description of mechanical effects: cavitation and role of bubbles;

ii. Factors that produce cavitation:

A. Pressure (compressional, rarefactional);

B. Frequency;

C. Beam focusing;

D. Pulsed/continuous;

E. Standing waves;

F. Boundaries;

G. Type of material and ambient conditions.

iii. Types of cavitation:

A. Stable and inertial cavitation;

B. Microstreaming;

C. Nucleation sites.

iv. Threshold phenomena for different types of tissues;

v. Bioeffects data on animals (lung hemorrhage, intestinal hemorrhage).

d. 3.4.2.4. Benefits of ultrasound versus risk: 
i. Benefits of use;

ii. Risk of use;

iii. Risk from not using ultrasound;

iv. Increase in risk as acoustic output increases;

v. Increase in diagnostic information as acoustic output increases;

vi. Increase in responsibility for user at higher output levels;

vii. The ALARA principle:

A. Controlling energy;

B. Controlling exposure time;

C. Controlling scanning technique;

D. Controlling system setup;

E. Effects of system capabilities;

F. Effects of operating mode (learn to distinguish);

G. Effects of transducer capabilities.

e. 3.4.2.5. The ODS:

i. Purpose: to display exposure indices;

ii. Ml;

iii. TI:
A. TIS;
B. TIB;
C. TIC

iv. Thresholds for display of indices:

A. eg, if system can exceed Tl or Ml of 1 .

v. System display levels:

A. eg, minimum TI displayed, minimum MI displayed, display increments.

vi. Explanation of the meaning of $\mathrm{TI}$ and $\mathrm{Ml}$ :

A. Threshold bioeffect levels vary depending on tissue type;

B. Bioeffect levels vary depending on frequency and pressure;

f. 3.4.2.6 Practicing the ALARA principle:

i. How to implement ALARA by using the TI and MI indices;

ii. Knowledge of system controls versus acoustic output:

A. Overall gain and time-gain compensation versus increasing output;

B. Dynamic range and postprocessing versus increasing output.

iii. Knowledge of system applications versus output:

A. Selection of appropriate range for task.

iv. Knowledge of transducer effects on output:
A. Frequency;
B. Focusing;
C. Pulse length;
D. Dwell time (scanned versus unscanned).

v. Knowledge of system operating mode versus output:
A. B-mode;
B. Doppler (spectral, color flow, amplitude Doppler);
C. M-mode;
D. Control exposure time;
E. Use of the minimum possible to obtain information.

vi. Clinical application examples: which indices are most important?
A. Fetal, cranial;
B. Fetal, Doppler;
C. Adult, thyroid;
D. Adult, carotid Doppler. 


\section{References}

1. National Council on Radiation Protection and Measurements. Exposure Criteria for Medical Diagnostic Ultrasound, II: Criteria Based on All Known Mechanisms. Bethesda, MD: National Council on Radiation Protection and Measurements; 2002. NCRP report 140.

2. British Medical Ultrasound Society Safety Group. Guidelines for the Safe Use of Diagnostic Ultrasound Equipment. London, England: British Medical Ultrasound Society; 2000.

3. European Committee of Medical Ultrasound Safety. Clinical Safety Statement for Diagnostic Ultrasound. London, England: European Committee of Medical Ultrasound Safety; 2006.

4. American Institute of Ultrasound in Medicine. How to interpret the ultrasound output display standard for higher acoustic output diagnostic ultrasound devices: version 2 [technical bulletin]. J Ultrasound Med 2004; 23:723-726.

5. US Department of Health and Human Services, Food and Drug Administration, Center for Devices and Radiological Health. Information for Manufacturers Seeking Marketing Clearance of Diagnostic Ultrasound Systems and Transducers. Washington, DC: US Department of Health and Human Services, Food and Drug Administration, Center for Devices and Radiological Health; 2008. http://www.fda.gov/cdrh/ode/guidance/560.pdf.

6. American Institute of Ultrasound in Medicine, National Electrical Manufacturers Association. Standard for RealTime Display of Thermal and Mechanical Acoustic Output Indices on Diagnostic Ultrasound Equipment. Revision 2. Laurel, MD: American Institute of Ultrasound in Medicine; Rosslyn, VA: National Electrical Manufacturers Association; 2004.

7. American Institute of Ultrasound in Medicine. Medical Ultrasound Safety. Laurel, MD: American Institute of Ultrasound in Medicine; 1994.

8. Szabo TL. Diagnostic Ultrasound Imaging: Inside Out. Burlington, MA: Elsevier Academic Press; 2004.

9. American Institute of Ultrasound in Medicine, National Electrical Manufacturers Association. Acoustic Output Measurement Standard for Diagnostic Ultrasound Equipment. Revision 1. Laurel, MD: American Institute of Ultrasound in Medicine; Rosslyn, VA: National Electrical Manufacturers Association; 2004. NEMA standards publication UD 2-2004.

10. American Institute of Ultrasound in Medicine. Introduction. In: Mechanical Bioeffects From Diagnostic Ultrasound: AIUM Consensus Statements. J Ultrasound Med 2000; 19(special issue):69-72.

11. American Institute of Ultrasound in Medicine. Section 1: conclusions and recommendations. In: Mechanical Bioeffects From Diagnostic Ultrasound: AlUM Consensus Statements. J Ultrasound Med 2000; 19(special issue): 73-76.
12. American Institute of Ultrasound in Medicine. Section 2: definitions and description of nonthermal mechanisms. In: Mechanical Bioeffects From Diagnostic Ultrasound: AlUM Consensus Statements. J Ultrasound Med 2000; 19(special issue):77-84.

13. American Institute of Ultrasound in Medicine. Section 3: selected biological properties of tissues: potential determinants of susceptibility to ultrasound-induced bioeffects. In: Mechanical Bioeffects From Diagnostic Ultrasound: AlUM Consensus Statements. J Ultrasound Med 2000; 19(special issue):85-96.

14. American Institute of Ultrasound in Medicine. Section 4: bioeffects in tissues with gas bodies. In: Mechanical Bioeffects From Diagnostic Ultrasound: AlUM Consensus Statements. J Ultrasound Med 2000; 19(special issue): 97-108.

15. American Institute of Ultrasound in Medicine. Section 5: nonthermal bioeffects in the absence of well-defined gas bodies. In: Mechanical Bioeffects From Diagnostic Ultrasound: AIUM Consensus Statements. J Ultrasound Med 2000; 19(special issue):109-119.

16. American Institute of Ultrasound in Medicine. Section 6: mechanical bioeffects in the presence of gas carrier ultrasound contrast agents. In: Mechanical Bioeffects From Diagnostic Ultrasound: AIUM Consensus Statements. J Ultrasound Med 2000; 19(special issue):120-142.

17. American Institute of Ultrasound in Medicine: Section 7: discussion of the mechanical index and other exposure parameters. In: Mechanical Bioeffects From Diagnostic Ultrasound: AIUM Consensus Statements. J Ultrasound Med 2000; 19(special issue):143-148.

18. American Institute of Ultrasound in Medicine. Section 8: clinical relevance. In: Mechanical Bioeffects From Diagnostic Ultrasound: AIUM Consensus Statements. J Ultrasound Med 2000; 19(special issue):149-168.

19. Church CC, Carstensen EL, Nyborg WL, Carson PL, Frizzell LA, Bailey MR. The risk of exposure to diagnostic ultrasound in postnatal subjects: nonthermal mechanisms. J Ultrasound Med 2008; 27(special issue):565-592.

20. Stratmeyer ME, Greenleaf JF, Dalecki D, Salvesen KA. Fetal ultrasound: mechanical effects. J Ultrasound Med 2008; 27(special issue):597-605.

21. Miller DL, Averkiou MA, Brayman AA, et al. Bioeffects considerations for diagnostic ultrasound contrast agents. J Ultrasound Med 2008; 27(special issue):611-632.

22. O'Brien WD, Deng CX, Harris GR, et al. The risk of exposure to diagnostic ultrasound in postnatal subjects: thermal effects. J Ultrasound Med 2008; 27(special issue):517535.

23. Abramowicz JS, Barnett SB, Duck FA, Edmonds PD, Hynynen $\mathrm{KH}$, Ziskin MC. Fetal thermal effects of diagnostic ultrasound. J Ultrasound Med 2008; 27(special issue):541-559.

24. Church CC, Miller MW. Quantification of risk from fetal exposure to diagnostic ultrasound. Prog Biophys Mol Biol 2007; 93:331-353. 


\section{Ultrasound Biosafety for the Sonographer and Sonologist}

25. World Federation for Ultrasound in Medicine and Biology WFUMB Symposium on Safety of Ultrasound in Medicine. Conclusions and recommendations on thermal and nonthermal mechanisms for biological effects of ultrasound; Kloster-Banz, Germany; 14-19 April, 1996. Ultrasound Med Biol 1998; 24(suppl 1):i-xvi, S1-S58.

26. Rados C. FDA cautions against ultrasound "keepsake" images. FDA Consumer 2004; January-February. http:// www.fda.gov/fdac/features/2004/104_images.html.

27. Sheiner E, Freeman J, Abramowicz JS. Acoustic output as measured by mechanical and thermal indices during routine obstetric ultrasound examinations. J Ultrasound Med 2005; 24:1665-1670.

28. Sheiner E, Shoham-Vardi I, Pombar X, Hussey MJ, Strassner HT, Abramowicz JS. An increased thermal index can be achieved during Doppler studies in obstetric sonography. J Ultrasound Med 2007; 26:71-76.

29. American Institute of Ultrasound in Medicine. AlUM practice guideline for the performance of an antepartum obstetric ultrasound examination. J Ultrasound Med 2003; 22:1116-1125.

30. Abramowicz JS, Fowlkes JB, Skelly AC, Stratmeyer ME, Ziskin MC. Conclusions regarding epidemiology for obstetric ultrasound. J Ultrasound Med 2008; 27(special issue): 637-644.

31. Fowlkes JB; Bioeffects Committee of the American Institute of Ultrasound in Medicine. American Institute of Ultrasound in Medicine consensus report on potential bioeffects of diagnostic ultrasound: executive summary. J Ultrasound Med 2008; 27(special issue):503-515.

32. Sheiner E, Shoham-Vardi I, Abramowicz JS. What do clinical users know regarding safety of ultrasound during pregnancy? J Ultrasound Med 2007; 26:319-325. 